\title{
Effectiveness of the physical activity promotion programme on the quality of life and the cardiopulmonary function for inactive people: Randomized controlled trial
}

\author{
Rocío Martín-Valero ${ }^{1 *}$, Antonio Ignacio Cuesta-Vargas ${ }^{1,2}$ and María Teresa Labajos-Manzanares ${ }^{1}$
}

\begin{abstract}
Background: The purpose is to assess cardiopulmonary function outcomes and quality of life values in inactive people that participated in the Physical Activity Promotion Programme (PAPP) against the control group that did not perform this program.

Method: A total of 100 subjects of both genders participated in the randomized controlled trial with systematic random sampling; all were aged 55 and older, from Torremolinos, Spain. Participants either received $(n=50)$ the PAPP for 60 minutes, twice a week during three months or $(n=50)$ they received health education. The effectiveness of the intervention was measured by general state of health the Short Form 12 health survey questionnaire, and the quality of life was determined with the EuroQoL-5D questionnaire. Cardiopulmonary function was measured with a spirometry and a walking test according to the Bruce protocol.

Results: This pilot study had a significant impact on the quality of life $(p=0.05)$ in men, which increased. However, the quality of life in women did not improve. The average changes in the lung and cardiovascular function was not significant between groups.

Conclusions: Changes in the quality of life measured with EQ-5D in the group of men who carried out the PAPP were statistically significant when comparing between groups. However changes in cardiopulmonary function were not as relevant when comparing between groups. There was a significant effect within each group in the pulmonary outcomes of values in men, within the experimental group.
\end{abstract}

Trial registration: Developed by the University of Málaga. ClinicalTrials.gov ID: NCT01172483.

Keywords: Promotion physical activity, Cardiopulmonary, Quality of life

\section{Background}

Prospective observational studies have suggested that inactive people have more death risk due to no specific cause and from specific diseases (e.g., cardiovascular disease, diabetes, obesity and others) associated with physical inactivity [1]. There is evidence that regular physical activity contributes to the primary and secondary prevention of several chronic diseases and is associated with a reduced risk of premature death [2].

\footnotetext{
*Correspondence: rovalemas@gmail.com

'Department of Physiotherapy, Faculty of Health Sciences, University of Málaga, Av/ Martiricos s/n, Málaga 29009, Spain

Full list of author information is available at the end of the article
}

Health-related quality of life is an important measure of the effect of an intervention program on cardiovascular disease [3]. There are studies in inactive people which have observed a decrease in lung function [4]. Forced expiratory volume in one second $\left(\mathrm{FEV}_{1}\right)$ was used in the cohort study of Buffalo as a predictor of survival and as a tool to assess the overall health of the population [5].

Epidemiological studies have showed that physical activity has a protective effect towards cardiovascular disease [6]. The physical activity helps to regulate the blood pressure [7]. There are previous studies about the physician's role in promoting physical activity [8]. However, it

\section{() Biomed Central}

(C) 2013 Martín-Valero et al.; licensee BioMed Central Ltd. This is an Open Access article distributed under the terms of the Creative Commons Attribution License (http://creativecommons.org/licenses/by/2.0), which permits unrestricted use, distribution, and reproduction in any medium, provided the original work is properly cited. 
is necessary to clarify the power of promoting physical activity for inactive people in the Primary Healthcare Centers.

The aim of the current study was to evaluate the effectiveness of the Physical Activity Promotion Programme (PAPP) on the quality of life and cardiopulmonary outcomes for the inactive subjects compared with people receiving education and advice in Primary Healthcare Centers. Therefore this study compares the observed changes in both the quality of life and cardiopulmonary outcomes for people who participated in the PAPP and those that did not carry out it.

\section{Methods}

\section{Study design and participants}

The design was a randomized controlled pilot clinical trial.

One hundred people from Primary Healthcare Centers in Málaga begun this research and seventy-five subjects completed the study. The age of the participants of both genders ranged between 57 and 69 .

\section{Inclusion and exclusion criteria}

The General Practitioner selected the participants who co-operated with the study. The criteria to be included in the study were inactive people, not engaged, with moderate physical activity for at least thirty minutes, five times a week [9]. Participants also had to have three or more of the following cardiovascular risk factors: increased blood pressure 140/90, smoking, cholesterol above $230 \mathrm{mg} / \mathrm{dl}$, a family member that had suffered a heart attack before the age of 55 if male or before the age of 65 if female and an obese or overweight (more than $8 \mathrm{~kg}$ ) insulin-dependent diabetic [10].

The criteria to be excluded from participation in the study were the following: infectious processes, malignancy, metastasis, osteoporosis, inflammatory arthritis or fractures, cognitive impairment due to any cause [11].

\section{Randomization}

The people who would participate in this study were randomized systematic procedures during the recruitment period. Random sequence generation was performed; people had to take a closed envelope from a box in order to form part of the intervention group or the control group. All the subjects who met the inclusion criteria had to carry out an initial evaluation before starting the procedure and at the end of twelve weeks the same procedure was carried out. The researcher, who did the evaluation, did not know what subjects would be assigned to each group.

\section{Procedure}

Physical activity promotion programme (PAPP)

The intervention group (IG) performed a PAPP twice a week for twelve weeks, following the criteria of the American College of Sports Medicine [9] in the Sports and Physical Medical Center in Torremolinos. An assigned professional health specialist carried out the procedure between October and March of 2010 and 2011. Each session lasted 60 minutes, and all protocols were developed for progressive intensity, depending on each person. The sessions were organized at the early stage of heating, followed by the aerobic phase and the cooling-stretch or final phase.

\section{Control group (CG)}

The control group received an educational health leaflet containing advice from Primary Healthcare Centers in Málaga, and continued with their daily routine activities. Subjects were assessed on the outcome measures at baseline, at twelve weeks follow-up by an independent blind assessor, at the Sports and Physical Medical Center in Torremolinos, Spain.

\section{Outcome measures}

The primary outcomes were the following: General Health Questionnaire determined with the Short Form-12 Health Survey (SF-12) and Health-related Quality of Life (HRQOL), determined with the EuroQoL-5D (EQ-5D).

The SF-12 questionnaire is a shortened version of the SF-36, and has a reliability coefficient of 0.97 [12]. We report the results of eight general health dimensions: physical functioning, physical role, body pain, general health, vitality, social functioning, emotional role and mental health [13]. These eight dimensions can also be used to generate both physical and mental health summary scores [12]. The SF-12 questionnaire will prove to be a practical alternative to the SF-36, in order to measure the overall health of the population, because of the high degree of correspondence between estimated physical and mental health measures, using the SF-12 and SF-36 questionnaires [14].

The EQ-5D has five domains: mobility, self-care, usual activities, pain, and anxiety/depression [15]. Each domain has three possible levels indicating; no problems, moderate problems or severe problems [16]. The EQ-5D valuation questionnaire comprises a visual analogue scale which was not included in this research. It has shown to be a acceptable and valid tool, with an average estimation of 0.87 [17].

The secondary outcomes were cardiopulmonary. The cardiac outcomes were at resting heart rate, and achieved at the end of the test. Subjects were questionnaire about the rate perceived effort (RPE) several times during the exercise test [18]. The pulmonary outcomes 
were the Tiffenau index, which is the ratio between forced expiratory volume in one second and forced vital capacity $\left(\mathrm{FEV}_{1} / \mathrm{FVC}\right)$, forced vital capacity $(\mathrm{FVC})$ and forced expiratory volume in one second $\left(\mathrm{FEV}_{1}\right)$. The values were expressed in absolute terms in milliliters and as percentage of theoretical value for individuals of the same age, weight and height in the reference population.

\section{The exercise test}

Subjects performed an exercise test on a treadmill according to the Bruce protocol [19]. The sub maximal protocol was used for the exercise test in which the subject would be over $85 \%$ of the maximum frequency. The test was brought to an end when the subject reached the maximum achievable intensity, taking into account the following criteria: the theoretical maximum heart rate according to age, usually calculated as two hundred twenty minus age in years.

Participants were asked to identify their perception of perceived exertion every thirty seconds and end the test with the scale of effort adapted by Borg (RPE) from zero to ten points [18]. The scale of the effort perception was defined as the subjective intensity of effort, stress, discomfort and / or fatigue you had felt during exercise [18].

The heart rate was measured at the beginning and at the end of the exercise test, which was called "HRf", and was obtained at the end of the running test on the treadmill.

\section{Spirometry}

The simple spirometry was used to measure lung outcomes with pneumotachograph Fleisch DATOSPIR 120 according to the SEPAR's criteria [20]. Three maneuvers were performed in order to get only the best values for the analysis. The values are determined with forced vital capacity (FVC), forced expiratory volume in one second $\left(\mathrm{FEV}_{1}\right)$, Tiffenau index which is the ratio between forced expiratory volume in one second and forced vital capacity $\left(\mathrm{FEV}_{1} / \mathrm{FVC}\right)$. The values are expressed in absolute terms in milliliters and as percentage of the theoretical value for individuals of the same age, weight and height in the reference population.

\section{Data collection}

All participants received information about the research and gave their written consent before participation. We performed a general clinical interview which included an exercise test and a spirometry.

\section{Data management and analysis}

All analyses were conducted using SPSS version 17.0. Kolmogorov-Smirnov tests were used to analyze the normality of the data distribution. Student's T tests for relational samples were performed to get the differences within each group after the intervention. We also used Student's $T$ test for independent samples to get the intergroup effect of this intervention.

\section{Sample size}

The sample size was calculated with an alpha error of 0.05 , a power of 0.80 and a beta risk of 0.20 according to the effect size of EQ-5D that it was made according to Cohen's criteria [21]. One hundred subjects took part in this research against 60 individuals are needed in a priori estimation: 30 individuals in the IG and 30 in the CG.

\section{Evaluation of clinical relevance}

Cohen's criteria were taken into account in the analysis of the effect size values, his criteria determine that values below 0.2 are considered to have no effect, those between 0.2 and 0.5 a small effect, between 0.5 and 0.8 a medium effect, and those above 0.8 are considered to have a huge effect [21].

\section{Ethics}

This study was authorized by the Ethics and Research Committee of the "Costa del Sol" Health District. All participants gave written informed consent, confidentiality and anonymity were also preserved at all times and the principles of the Declaration of Helsinki were respected.

\section{Results}

The sample consisted of thirty-one men and forty-four women. The subjects mean aged was $62.28 \pm 6.9$ years. Gender difference is reflected in all data. The initial characteristic of the participants are shown in Table 1. Regarding the systolic and diastolic blood pressure at rest, the initial values of systolic blood pressure were greater than $140 \mathrm{~mm} \mathrm{Hg}$ (Table 1). The descriptive values of the changes within each group, obtained in the self-reported questionnaires and the cardiopulmonary outcomes by gender difference (Table 2).

In our study, the changes between both the experimental and the control group in the self-reported questionnaires found the range of EQ-5D of the male population with a difference between groups in the effect size value of $0.05(\mathrm{p}=0.05)$, whereas women did not improve their quality of life. The changes between both the experimental and control group in the cardiopulmonary function outcome for the $\mathrm{FEV}_{1} / \mathrm{FVC}$ value did not show statistically significant changes in the experimental group, which the mean $\mathrm{FEV}_{1} / \mathrm{FVC}$ value for both 
Table 1 Initial characteristic according the gender difference

\begin{tabular}{|c|c|c|c|c|}
\hline & \multicolumn{2}{|c|}{ Control group } & \multicolumn{2}{|c|}{ Intervention group } \\
\hline & Men mean $(\mathrm{Cl})$ & Women mean $(\mathrm{Cl})$ & Men mean $(\mathrm{Cl})$ & Women mean $(\mathrm{Cl})$ \\
\hline age (years) & 64.25 (59-69) & $62.82(60-65)$ & $60.50(57-63)$ & $63.26(60-66)$ \\
\hline Weight (Kg) & 93.29(79-106) & $78.50(71-85)$ & $87.44(77-97)$ & $78.21(71-85)$ \\
\hline Height (metre) & $1.68(1.64-1.72)$ & $1.58(1.5-1.60)$ & $1.67(1.62-1.74)$ & $1.56(1.54-1.59)$ \\
\hline $\mathrm{SPB}(\mathrm{mm} \mathrm{Hg})$ & 156(143-168) & 133(127-140) & 144(134-154) & 131(125-137) \\
\hline DPB (mm Hg) & $83(76-91)$ & $78.91(75-82)$ & 79(69-89) & $80.21(76-85)$ \\
\hline $\mathrm{HRr}(\mathrm{B} / \mathrm{min})$ & $73(66-80)$ & 76.18(72-80) & $75.17(68-82)$ & $80.04(74-86)$ \\
\hline HRmax (B/min) & 132(127-136) & 133.43(131-135) & 134(130-138) & 132.57(129-136) \\
\hline $\mathrm{HRf}(\mathrm{B} / \mathrm{min})$ & 122(114-130) & 125(118-132) & 124(115-132) & $123.21(115-131)$ \\
\hline RPE & $5.75(4.7-6.8)$ & $5.27(4.50-6.04)$ & $5.28(4.44-6.11)$ & $5(4.42-5.58)$ \\
\hline FVC $(\mathrm{L})$ & $3.78(2.9-4.6)$ & $2.76(2.21-3.3)$ & $3.88(3.14-4.62)$ & $2.54(2.22-2.86)$ \\
\hline $\mathrm{FEV}_{1}(\mathrm{~L})$ & $2.55(1.94-3.16)$ & $1.68(1.32-2.03)$ & 2.44(1.99-2.9) & $1.77(1.51-2.02)$ \\
\hline $\mathrm{FEV}_{1} / \mathrm{FVC}(\%)$ & 66.59(57-76) & 68(59-78) & $65.61(57-74)$ & $71(63-79)$ \\
\hline EQ-5D (0-1) & $0.57(0.38-0.75)$ & $0.59(0.48-0.72)$ & $0.58(0.36-0.76)$ & $0.53(0.40-0.67)$ \\
\hline Physical role (0-100) & $46(40-51)$ & $44.32(40-49)$ & $48(43-53)$ & $42.5(37-48)$ \\
\hline Social (0-100) & $26.27(26.3-26.3)$ & $31.71(23-40)$ & $31.3(20.22-42.4)$ & $25.15(18-32)$ \\
\hline Vitality (0-100) & $52.8(46-60)$ & $49(43-55)$ & $46.1(40-52)$ & $51(44-57)$ \\
\hline Mental (0-100) & $34.06(26-42)$ & $36.43(30-42)$ & $37.5(30-44)$ & $39(33-45)$ \\
\hline
\end{tabular}

$\mathrm{Cl}$ : confidence interval.

SPB: systolic blood pressure.

$\%$ : percentage.

L: liter.

$\mathrm{B} / \mathrm{min}$ : beats per minute.

$\mathrm{HRr}$ : heart rate on resting.

DPB: diastolic blood pressure.

HRf: heart rate at the end.

HRmax: maximum heart rate.

RPE: rate perceived effort.

men and women was $(-10.45$ vs. $5.17 \%)$ respectively (Table 2). The men of the control group showed a greater negative effect ( -17.48 vs. $-1.23 \%)$, compared to women with a smaller negative effect (Table 2).

\section{Discussion}

Quality of life improved significantly for inactive subjects who carried out the PAPP $(\mathrm{p}=0.05)$. Besides, pulmonary function outcomes $\mathrm{FVC}$ and $\mathrm{FEV}_{1} / \mathrm{FVC}$ values found statistically significant differences within experimental group. However, these differences were not found within the control group. Other clinical trials with community intervention programs have increased the level of physical activity, without improving the level of the quality of life [22]. In contrast, others have improved the quality of life without changing the level of physical activity [23]. Statistically differences were observed for people with depression after the DeLLITE program of physical activity in the quality of mental health, measured with the SF-36 questionnaire [24].

In previous research, the criteria established for the minimum important differences of the ranged of EQ-5D from 0.033 [25] to 0,074 [26]. In the current study, the male population was within this range of EQ-5D with a difference between groups in the effect size value of 0.05 $(p=0.05)$, whereas women did not improve their quality of life. In cardiac patients an effect size of 0.31 , $(\mathrm{p}=0.001)$ was observed in the quality of life with a positive statistical significance [27]. However, a crosssectional study showed a decreased quality of life value with respect to the EQ-5D questionnaire, in the people aged between 66-79 years with sedentary lifestyles [28]. Furthermore, it is necessary to reach the consensus about the clinically important difference for EQ-5D.

According to the general health status which was determined with the SF-12 questionnaire there were no statistically significant changes between groups, after the intervention of the PAPP, as the current study has shown. However, other studies showed improved cognitive function (effect size 1.17) in people who performed physical activity [29]. Like as in other studies, they found that active subjects had fewer psychological problems than inactive subjects [30]. Statistically also changes in the SF-36 questionnaire had been observed after performing a program of physical activity in water during two months. These improvements were obtained in all 
Table 2 Changes within each group obtained in the cardiopulmonary outcomes and the questionnaires according gender difference

\begin{tabular}{|c|c|c|c|c|}
\hline & & & Interve & oup \\
\hline & Men mean $(\mathrm{Cl})$ & Women mean $(\mathrm{Cl})$ & Men mean $(\mathrm{Cl})$ & Women mean $(\mathrm{Cl})$ \\
\hline Weight (Kg) & $1.95(0.47-3.44)^{* *}$ & $-0.63(-2.19-0.92)$ & $-0.05(-3.13-3.02)$ & $-0.50(-1.82-0.82)$ \\
\hline SPB (mm Hg) & $13.08(0.53-25.63)^{*}$ & $-3.13(-8.73-2.46)$ & $-4.33(-20-11.44)$ & $-4.65(-10.27-1.41)$ \\
\hline DPB (mm Hg) & $0.83(-4.83-6.49)$ & $-0.04(2.40-2.31)$ & $-4.50(-16-7.08)$ & $1.43(-2.93-5.80)$ \\
\hline $\operatorname{HRr}(B / \min )$ & $-3.75(-9.86-2.36)$ & $-4.5\left(-8.45-(-0.54)^{*}\right.$ & $-2.16(-5.80-1.47)$ & $1.04(-2.64-4.72)$ \\
\hline HRmax (B/min) & $-0.58(-1.86-0.70)$ & $-0.09(-0.32-0.142)$ & $-0.42(-1.23-0.40)$ & $-1.78(-4.62-1.05)$ \\
\hline HRfinal (B/min) & $4(-1.92-9.92)$ & $1.72(-4.75-8.21)$ & $1(-6.01-8.01)$ & 3.82(-4.09-11.74) \\
\hline RPE & $0.83(-0.13-1.80)$ & $0.91(0.22-1.59)^{* *}$ & $0.22(-0.60-1.05)$ & $0.39(-0.29-1.07)$ \\
\hline FVC (L) & $0.82(0.33-1.31)$ & $0.38(-0.09-0.85)$ & $0.73(0.18-1.27)^{* *}$ & $0.10(-0.06-0.27)$ \\
\hline FEV1 (L) & $0.06(-0.38-0.51)$ & $-0.02(-0.24-0.19)$ & $0.11(-0.03-0.26)$ & $-0.07(-0.23-0.08)$ \\
\hline FEV1/FVC (\%) & $17.47(-28.01-(-6.95)$ & $-1.22(-11.30-8.85)$ & $-10.45\left(-18.6-(-2.23)^{* *}\right.$ & $5.17(-11.45-1.11)$ \\
\hline EQ-5D (0-1) & $-0.24(-0.43-0.043)^{*}$ & $0.065(-0.166-0.03)$ & $0.02(-0.18-0.23)$ & $0.14(-0.30-0.01)$ \\
\hline Physical role (0-100) & $0(-7.27-7.27)$ & $3.43(-1.14-8.01)$ & $1.16(-5.12-8.34)$ & $4.29(-0.619-9.21)$ \\
\hline Social (0-100) & $10.09(-8.45-28.65)$ & $1.12(-12.58-14.81)$ & $10.09(-8.45-28.65)$ & $8.07(-21.81-5.65)$ \\
\hline Body Pain (0-100) & $7.64(-0.46-15.75)^{*}$ & $8.33(0.95-15.72)^{*}$ & $5.09(-4.26-14.45)$ & $5.09(-4.26-14.45)$ \\
\hline General Health (0-100) & $1.23(-2.69-5.16)$ & $0.43(-3.18-2.32)$ & $3.50(-7.58-0.58)$ & $0.95(-3.29-1.37)$ \\
\hline Vitality(0-100) & $8.51(-1.67-18.70)$ & $O(-5.11-5.11)$ & $2.87(-5.15-10.90)$ & $2.68(-5.32-10.69)$ \\
\hline Mental(0-100) & $2.03(-9.17-13.23)$ & $3.04(-15.42-9.32)$ & $1.74(-10.31-13.79)$ & $6.70(-3.03-16.44)$ \\
\hline
\end{tabular}

** $p=0.01$.

$\mathrm{Cl}$ : confidence interval.

SPB: systolic blood pressure.

RPE: rate perceived effort.

${ }^{*} p=0.05$.

$\mathrm{HRr}$ : heart rate on resting.

HRf: heart rate at the end.

HRmax: maximum heart rate.

$\mathrm{B} / \mathrm{min}$ : beats per minute.

$\%$ : percentage.

$\mathrm{FEV}_{1}$ : forced expiratory volume.

$\mathrm{FEV}_{1} /$ FVC: Tiffenau index.

FVC: forced vital capacity.

L: liter.

the fields of the quality of life, except in the emotional role and in general health [31].

Regarding the systolic and diastolic blood pressure, we did not observe statistically significant differences in the effect size on diastolic blood pressure after the procedure. In contrast, the effect size for cardiac subjects showed statistically better improvement $(\mathrm{p}=0.01)$ [27]. In a recent meta-analysis, both resistance training programs and isometric hand grip could help decrease blood pressure [32]. Besides, resistance programs could decrease the risk of cardiovascular disease [33].

There were a slight decrease in systolic blood pressure (SDP) at rest concerning the same value in both men and women in the experimental group (-4.33 vs. -4 $.65 \mathrm{Mm} \mathrm{Hg}$ ). However, men in the control group showed an increase systolic blood pressure (13.09 vs. $-3.13 \mathrm{Mm} \mathrm{Hg}$, $\mathrm{p}=0.05)$ compared with women who showed a decrease in systolic blood pressure. In contrast, people with mild hypertension found a significant decrease in systolic blood pressure, as a result of performing a program of low intensity running [34].

Respect to diastolic blood pressure (DBP) not statistically significant changes were found within each group. There was a decrease in DBP in men in the experimental group ( -4.5 vs. $1.43 \mathrm{~mm} \mathrm{Hg}$ ) versus a slight increase in DBP in women (Table 2). However, people with hypertension got statistically significant decrease after receiving a program of low intensity exercise [34]. Women with metabolic syndrome also got decrease both blood pressures when performed a program of low intensity [35]. Besides, a decrease in blood pressure has been observed after performed a program of moderate intensity resistance exercises [36].

According to the cardiac outcomes not statistically significant changes were found between both the experimental and control group. However, we found statistically significant changes in resting HR in the control group of women. The mean HR value was $(-3.75$ vs. $-4.5, \mathrm{p}=0.05)$ 
in men and women respectively. On the other hand, statistically significant changes were not observed in the experimental group (Table 2). There are studies that found differences in the cardiac function depending on the amount of body fat of the subject [37]. The Fit \& Firm program showed significant improvement in heart rate [38]. It could be possible that the cardiac function response depends on a mix of genetic factor and the intensity of training.

The pulmonary outcomes except $\mathrm{FEV}_{1}$ improved significantly $(\mathrm{p}<0.01)$ in men of the experimental group. In a recent study, all pulmonary function parameters except $\mathrm{FEV}_{1} / \mathrm{FVC}$ improved significantly $(\mathrm{p}<0.0001)$ in both yoga and swimming groups [39]. Besides, better pulmonary functions in subjects performing yoga as well as swimming are documented $[4,40]$. Statistically significant changes in pulmonary function of $\mathrm{FEV}_{1} / \mathrm{FVC}$ and $\mathrm{FEV}_{1}$ values in people who performed regularly physical activity compared with sedentary people were shown [4]. Apart from that, the participants performed a four-week program who found statistically significant changes in $\mathrm{FEV}_{1}$ and $\mathrm{FEV}_{1} / \mathrm{FVC}$ (3.96 vs. 0.96, $\mathrm{p}=0.001$ ), but there were no statistically significant changes in the FVC value (4.13, $\mathrm{p}=0.43$ ) [39]. This program included upper limb resistive exercises for thirty minutes, supplemented with ten minutes of breathing exercises [39]. Nevertheless, the program "Exercise on Prescription" not found effect on the pulmonary outcome in the physical inactive women in the Netherlands [41].

Type II error should take into account the outcomes which did not show effects due to lack of subjects. Future studies will be necessary to find the reason to explain the difference in $\mathrm{FVC}$ and $\mathrm{FEV}_{1} / \mathrm{FVC}$ outcomes between men and women [5].

\section{Conclusions}

Changes statistically significant in the quality of life measured with EQ-5D in the group of men who carried out the PAPP when comparing between groups. However changes in cardiopulmonary function were not as relevant when comparing between groups. On the contrary, there was a significant effect within each group in the pulmonary outcomes of values in men, within the experimental group.

To ensure the quality of this randomized clinical trial the guide developed by the CONSORT statement (Consolidated Standards of Reporting Trials) [42] has been followed. ClinicalTrials.gov ID: NCT01172483.

\section{Competing interests}

The authors declare that they have no competing interests.

\section{Authors' contributions}

All authors have made significant contributions to the article. AICV coordinated the project and contributed to the conception and design of this study. RMV and AICV were responsible of the acquisition, analysis and interpretation of data, both contributed to the screened articles and drafted the manuscript. AICV and MTL made substantial contributions to the critical revision of the paper. All authors read and approved the final manuscript.

\section{Acknowledgements}

This research was made possible by a grant received as a research fellow from the Junta de Andalucía and the University of Málaga. It was partially funded by grants from the OTRI-UMA (8.06/5.42.3064-2). We would like to thank the subjects who participated in this research, Primary Care Team and the Sports Center in Torremolinos.

\section{Author details}

${ }^{1}$ Department of Physiotherapy, Faculty of Health Sciences, University of Málaga, Av/ Martiricos s/n, Málaga 29009, Spain. ${ }^{2}$ School of Clinical Science at Queensland University of Technology, Level 6, O Block, D WingKelvin Grove, Brisbane, Australia.

Received: 7 September 2012 Accepted: 4 February 2013

Published: 12 February 2013

\section{References}

1. Haskell WL, Blair SN, Hill JO: Physical activity: health outcomes and importance for public health policy. Prev Med 2009, 49(4):280-282.

2. Warburton DE, Nicol CW, Bredin SS: Health benefits of physical activity: the evidence. CMAJ 2006, 174(6):801-809.

3. Lam CL, Lauder IJ: The impact of chronic diseases on the health-related quality of life (HRQOL) of Chinese patients in primary care. Fam Pract 2000, 17(2):159-166.

4. Prakash S, Meshram S, Ramtekkar U: Athletes, yogis and individuals with sedentary lifestyles; do their lung functions differ? Indian J Physiol Pharmacol 2007, 51(1):76-80.

5. Schunemann HJ, Dorn J, Grant BJ, Winkelstein W Jr, Trevisan M: Pulmonary function is a long-term predictor of mortality in the general population: 29-year follow-up of the Buffalo Health Study. Chest 2000, 118(3):656-664.

6. Lee IM: Physical activity and cardiac protection. Curr Sports Med Rep 2010, 9(4):214-219.

7. Scher LM, Ferriolli E, Moriguti JC, Scher R, Lima NK: The effect of different volumes of acute resistance exercise on elderly individuals with treated hypertension. J Strength Cond Res 2011, 25(4):1016-1023.

8. Brown WJ: Individual or population approaches to the promotion of physical activity. . . is that the question? J Sci Med Sport 2006, 9(1-2):35-37. discussion 38-9.

9. Haskell WL, Lee IM, Pate RR, Powell KE, Blair SN, Franklin BA, et al: Physical Activity and Public Health: Updated Recommendation for Adults From the American College of Sports Medicine and the American Heart Association. Circulation 2007, 116(9):1081-1093.

10. Weng C, Tu SW, Sim I, Richesson R: Formal representation of eligibility criteria: a literature review. J Biomed Inform 2010, 43(3):451-467.

11. Van Spall HG, Toren A, Kiss A, Fowler RA: Eligibility criteria of randomized controlled trials published in high-impact general medical journals: a systematic sampling review. JAMA 2007, 297(11):1233-1240.

12. Ware J, Kosinski M, Keller SD: A 12-Item Short-Form Health Survey: construction of scales and preliminary tests of reliability and validity. Med Care 1996, 34(3):220-233.

13. Jenkinson C, Layte R: Development and testing of the UK SF-12 (short form health survey). J Health Serv Res Policy 1997, 2(1):14-18.

14. Gandek B, Ware JE, Aaronson NK, Apolone G, Bjorner JB, Brazier JE, et al: Cross-validation of item selection and scoring for the SF-12 Health Survey in nine countries: results from the IQOLA Project, International Quality of Life Assessment. J Clin Epidemiol 1998, 51(11):1171-1178.

15. Herdman M, Badia X, Berra S: EuroQol-5D: a simple alternative for measuring health-related quality of life in primary care. Aten Primaria 2001, 28(6):425-430.

16. Papaioannou D, Brazier J, Parry G: How valid and responsive are generic health status measures, such as EQ-5D and SF-36, in schizophrenia? A systematic review. Value Health 2011, 14(6):907-920.

17. Jia H, Lubetkin El: Estimating EuroQol EQ-5D scores from Population Healthy Days data. Med Decis Making 2008, 28(4):491-499.

18. Borg GA: Psychophysical bases of perceived exertion. Med Sci Sports Exerc 1982, 14(5):377-381. 
19. Mahler DA, Froelicher VF, Miller NH, York TD: ACSM's Guidelines for Exercise Testing and Prescription. 5th edition. Baltimore, Md: Williams \& Wilkins; 1995

20. Miller MR, Hankinson J, Brusasco V, Burgos F, Casaburi R, Coates A, Crapo R et al: Standardisation of spirometry. Eur Respir J 2005, 26(2):319-338.

21. Cohen J: Statistical power analysis for the behavioral sciences; 1988. Available from: [http://www.questia.com/PM.qst?a=o\&d=98533106].

22. Kolt GS, Schofield GM, Kerse N, Garrett N, Oliver M: Effect of telephone counseling on physical activity for low-active older people in primary care: a randomized, controlled trial. J Am Geriatr Soc 2007, 55(7):986-992.

23. Kerse $N$, Elley $C R$, Robinson $E$, Arroll B: Is physical activity counseling effective for older people? A cluster randomized, controlled trial in primary care. J Am Geriatr Soc 2005, 53(11):1951-1956.

24. Kerse N, Hayman KJ, Moyes SA, Peri K, Robinson E, Dowell A, et al: Home-based activity program for older people with depressive symptoms: DeLLITE-a randomized controlled trial. Ann Fam Med 2010, 8(3):214-223.

25. Sullivan PW, Lawrence WF, Ghushchyan V: A national catalog of preference-based scores for chronic conditions in the United States. Med Care 2005, 43(7):736-749.

26. Walters SJ, Brazier JE: Comparison of the minimally important difference for two health state utility measures: EQ-5D and SF-36D. Qual Life Res 2005, 14(6):1523-1532.

27. Conn VS, Hafdahl AR, Moore SM, Nielsen PJ, Brown LM: Meta-analysis of interventions to increase physical activity among cardiac subjects. Int J Cardiol 2009, 133(3):307-320

28. Kostka T, Bogus K: Independent contribution of overweight/obesity and physical inactivity to lower health-related quality of life in communitydwelling older subjects. Z Gerontol Geriatr 2007, 40(1):43-51.

29. Angevaren M, Aufdemkampe G, Verhaar HJ, Aleman A, Vanhees L: Physical activity and enhanced fitness to improve cognitive function in older people without known cognitive impairment. Cochrane Database Syst Rev 2008, 3(3):CD005381.

30. Akbartabartoori M, Lean ME, Hankey CR: The associations between current recommendation for physical activity and cardiovascular risks associated with obesity. Eur J Clin Nutr 2008, 62(1):1-9.

31. Saavedra JM, De La Cruz E, Escalante Y, Rodriguez FA: Influence of a medium-impact aquaerobic program on health-related quality of life and fitness level in healthy adult females. J Sports Med Phys Fitness 2007, 47(4):468-474

32. Cornelissen VA, Fagard RH, Coeckelberghs E, Vanhees L: Impact of resistance training on blood pressure and other cardiovascular risk factors: a meta-analysis of randomized, controlled trials. Hypertension 2011, 58(5):950-958.

33. Kodama S, Saito K, Tanaka S, Maki M, Yachi Y, Asumi M, et al: Cardiorespiratory fitness as a quantitative predictor of all-cause mortality and cardiovascular events in healthy men and women: a meta-analysis. JAMA 2009, 301(19):2024-2035.

34. Hua LP, Brown CA, Hains SJ, Godwin M, Parlow JL: Effects of low-intensity exercise conditioning on blood pressure, heart rate, and autonomic modulation of heart rate in men and women with hypertension. Biol Res Nurs 2009, 11(2):129-143.

35. Roussel M, Garnier S, Lemoine S, Gaubert I, Charbonnier L, Auneau G, et al: Influence of a walking program on the metabolic risk profile of obese postmenopausal women. Menopause 2009, 16(3):566-575.

36. Fagard $\mathrm{RH}$, Cornelissen VA: Effect of exercise on blood pressure control in hypertensive patients. Eur J Cardiovasc Prev Rehabil 2007, 14(1):12-17.

37. Chantler PD, Clements RE, Sharp L, George KP, Tan LB, Goldspink DF The influence of body size on measurements of overall cardiac function. Am J Physiol Heart Circ Physiol 2005, 289(5):H2059-H2065.

38. King AC, Pruitt LA, Phillips W, Oka R, Rodenburg A, Haskell WL: Comparative effects of two physical activity programs on measured and perceived physical functioning and other health-related quality of life outcomes in older adults. J Gerontol A Biol Sci Med Sci 2000, 55(2):M74-M83.

39. Singh VP, Jani $H$, John V, Singh $P$, Joseley T: Effects of upper body resistance training on pulmonary functions in sedentary male smokers. Lung India 2011, 28(3):169-173.

40. Gupta SS, Sawane MV: A comparative study of the effects of yoga and swimming on pulmonary functions in sedentary subjects. Int $\mathrm{J}$ Yoga 2012, 5(2):128-133.
41. Gademan MG, Deutekom M, Hosper K, Stronks K: The effect of exercise on prescription on physical activity and wellbeing in a multi-ethnic female population: A controlled trial. BMC Public Health 2012, 12(1):758.

42. Atman DG, Schulz KF, Moher D, Egger M, Davodpff F, Elbourne D, CONSORT GROUP (Consolidated Standards of Reporting Trials), et al: The revised CONSORT statement for reporting randomized trials: explanation and elaboration. Ann Int Med 2001, 134(8):663-694.

doi:10.1186/1471-2458-13-127

Cite this article as: Martín-Valero et al.: Effectiveness of the physical activity promotion programme on the quality of life and the cardiopulmonary function for inactive people: Randomized controlled trial. BMC Public Health 2013 13:127.

\section{Submit your next manuscript to BioMed Central and take full advantage of:}

- Convenient online submission

- Thorough peer review

- No space constraints or color figure charges

- Immediate publication on acceptance

- Inclusion in PubMed, CAS, Scopus and Google Scholar

- Research which is freely available for redistribution

Submit your manuscript at www.biomedcentral.com/submit
C) BioMed Central 